\title{
Performance Evaluation of Multipath Routing Protocol (AOMDV) In Mobile Ad Hoc Networks
}

\author{
Geetha . $\mathrm{N}^{1}$ \\ \{sng.mca@psgtech.ac.in\} \\ Assistant Professor (Selection Grade), Department of Computer Applications, PSG College of \\ Technology, Coimbatore ${ }^{1}$
}

\begin{abstract}
Multiple pathways between the source and destination can be used to prevent link/node failure during the transmission of data packets from the source to the destination in a Mobile Ad hoc Network (MANET). In this paper, a systematic performance evaluation of Adhoc On Demand Multipath Distance Vector (AOMDV) routing protocol is carried out. The simulation is done in Network Simulator-2 (NS2) and the different metrics are used for performance analysis. The results obtained from the evaluation prove that the routing protocol performance varies from network to network depending upon the topology. A large variety of factors affect the performance of the protocol.
\end{abstract}

Keywords: Mobile Ad hoc Network (MANET), Network Simulator-2 (NS2), Adhoc On Demand Multipath Distance Vector (AOMDV), protocol.

\section{Introduction}

Mobile Ad hoc Network (MANET) is a decentralised autonomous wireless system that consists of a collection of nodes deployed in a monitoring environment. MANET is a selfconfigurable mobile mesh network. A mobile node network (MANET) is a group of mobile nodes that may communicate with each other via wireless communication devices. The nodes can be of any devices like laptops, personal computers, MP3 player, mobile phones etc. These nodes can be deployed in any place cyclone affected areas, tsunami affected locations or in automobiles or with people having small electronic devices. These nodes communicate with each other randomly forming a dynamic topology. Nodes communicate to each other and also act as router in forwarding packets to the neighbouring nodes. The self configuring nature of the nodes in the network adapts this to be more suitable for any disaster situation.

A performance analysis of AOMDV (Adhoc On Demand Multipath Distance Vector), a multipath routing protocol, [1] is carried out to evaluate the protocol and specify the situations where this protocol can be applied. This paper is organized into two parts. First, simulate the wireless network with 100, and analyze the performance by increasing the node density. Simulations are performed using NS-2 [5][7] and the transmissions are traced and maintained in a trace file. Scripting language AWK is used to gain useful information from the trace file and to calculate the QoS metrics and to perform analysis. Second, X-graph [7][8] is used to compare the performance of the protocol with varying node density.

\section{Related Work}


Routing protocols can be broadly classified into proactive, reactive, and hybrid protocols [3]. This section briefly describes the routing protocols in the literature.

Proactive (table-driven) routing protocols follow the principles of traditional datagram networks. These protocols adopt distance-vector or link-state routing strategies and any topology changes due to mobility of nodes are updated periodically throughout the network. The routing information is maintained in the routing tables for the unused paths as well. The main drawback is that routing tables maintain path details which are unused leading to routing overhead and may use maximum bandwidth if the network topology changes frequently.

Reactive (on-demand) routing protocols employs on demand approach. The routes are discovered by source nodes only on-demand. These protocols maintain routes only on demand and hence the network overhead is reduced when there is only fewroutes are active at any time. Low consumption of bandwidth is in reactive protocols than proactive protocols, but with a tradeof that the delay in finding a route is large. In reactive protocols, route discovery phase is invoked wherever there is a packet for transmission to the intended destination from the source. The main drawback of reactive protocol is the delay in first packet transmission. Another drawback is that the network traffic increases on route computation for highly dynamic network. Also packets may be lost during transmission due to dynamic topology changes. Reactive protocols are commonly used in network with less number of nodes.

To improve network efficiency and scalability, the hybrid routing protocol incorporates the benefits of both proactive and reactive techniques. If the topology is fixed this protocol gives a better performance. However, due to the change of position of the nodes, topology change occurs in MANET resulting in path changes. The topology based routing protocols have low throughput and less route convergence due to high mobility of node during simulation. From the analysis made, we infer that the position based routing protocols are identified as a more suitable routing protocols for highly dynamic network and to increase the scalability.

\section{Routing Technique}

Multipath routing is a routing technology wherein multiple paths are available between any two nodes yielding a better performance by improving fault tolerance, reliability and speed.

\section{A. On-demand Multipath Routing:}

Multipath routing protocol Ad hoc Ondemand Multipath Distance Vector (AOMDV). The AOMDV protocol is an upgraded version of the AODV protocol that includes route discovery and management as well as a routing table. During route discovery process, AOMDV protocol maintains routing information with a list of neighbour nodes with the hop counts for each destination. Each route holds unique sequence number to maintain the life of the route. Route advertisements are made which is the alternate route. The main advantage is that it allows intermediate node to respond RREQ message with RREP messages. The destination will respond with RREP messages for the RREQ message received from different neighbour nodes.

\section{B. Route discovery mechanism}




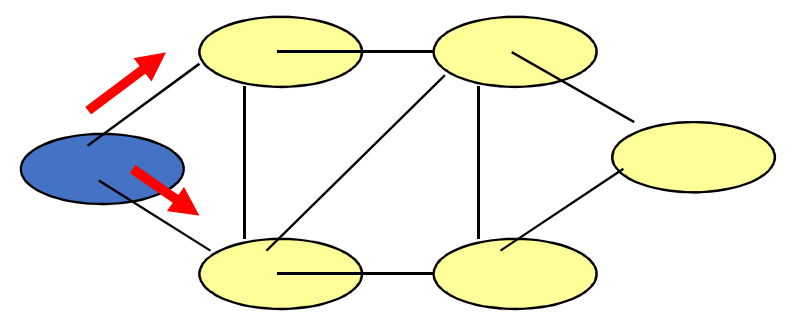

Fig. 1. Node ' $S$ ' broadcasting RREQ packet

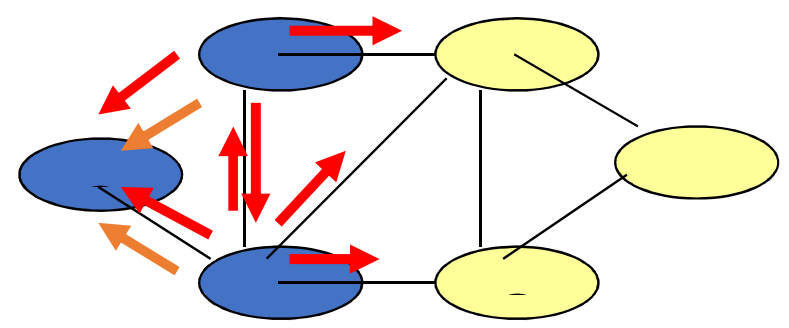

Fig. 2. Node ' $A$ ' and ' $B$ ' broadcasting RREQ packet and reverse path formation

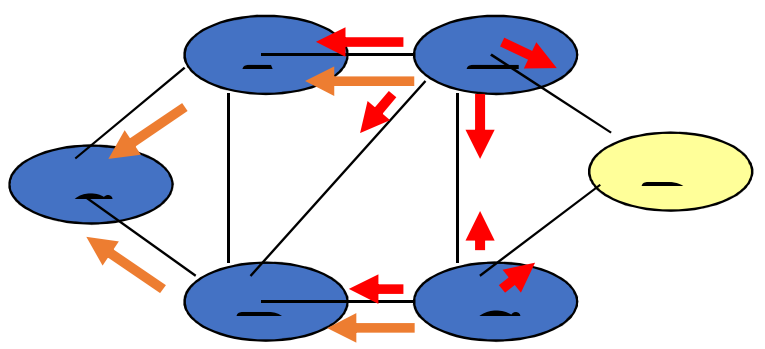

Fig. 3. Node ' $C$ ' and ' $E$ ' broadcasting RREQ packet and reverse path formation

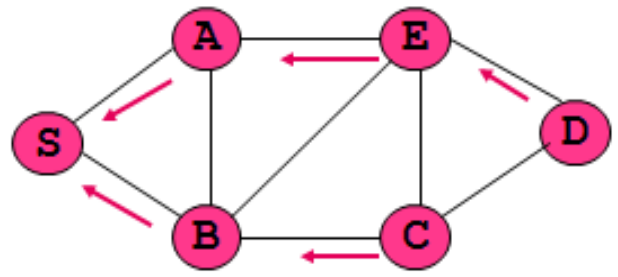

Fig. 4. Node 'D' sending RREP packet 


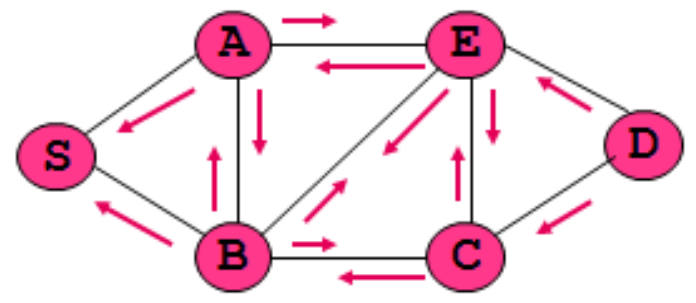

Fig. 5. Forming reverse path with duplicate RREQ packets also

The Fig 1 - Fig 5 represents route discovery process of AOMDV with 6 nodes. AOMDV protocol calculates different loop free and disjoint paths. To avoid loops, AOMDV protocol accepts RREQ from nodes with lower or equal sequence number and advertises only one path at a time.

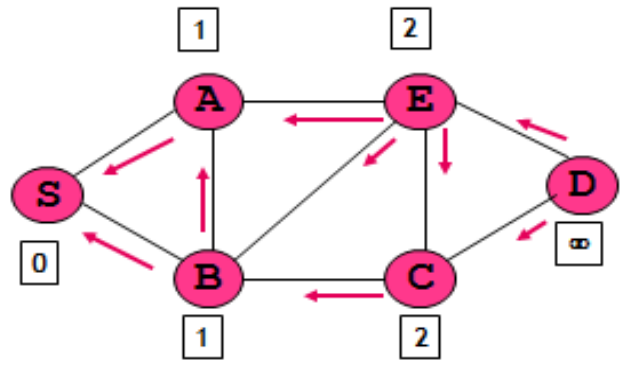

Fig. 6. Reverse path formation in AOMDV

\section{Simulation Environment}

Network simulator-2 (NS2)[5][6] is used to simulate the network. The packets are transferred using TCP transportation protocol and AOMDV routing protocol.

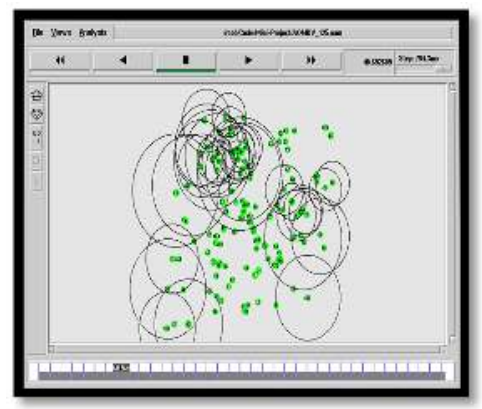

Fig. 7. Simulation of 100 nodes in NS2 and packet transfer using AOMDV 
The simulation of AOMDV protocol with 100 nodes is shown in fig. 7. Networks with 125, 150, 175 and 200 nodes are simulated in similar manner and their transmissions are traced and maintained.

\section{Performance Analysis}

The routing protocol is analyzed based on different metrics to assess the performance of the AOMDV[2][4] routing protocol. The simulations are carried out by varying node density and the results are analysed.

\section{Throughput:}

The throughput is the ratio of total data received from the transmitter to the total data received by the receiver in a certain time frame. Throughput can be shown as

Throughput $=($ Received data size $) /$ Total simulation time

The throughput of the network is affected due to unreliable communication, dynamic topology changes, limited bandwidth and energy of the nodes.

\section{End to end delay:}

The average time taken for a data packet to reach the destination is known as end-toend delay. The time includes the delay induced by the route discovery procedure as well as the time spent waiting in the delay queue while data packets are being transmitted. The data packets that are successfully reaching the destinations are counted. End to end delay is mathematically represented as End to End delay $=$ Sum of arrival time-sum of send time / number of connections

\section{Packet delivery ratio:}

Packet delivery ratio is the ratio of the number of packets sent to the destination successfully to the number of packets delivered from source. Packet delivery ratio can be mathematically given as Packet delivery ratio $=$ Number of packets received $/$ Total packets sent

\section{Residual energy:}

Residual energy can be defined as the energy left behind after the transmission of the packets between the nodes. The residual energy of nodes has strong connection with the node density.

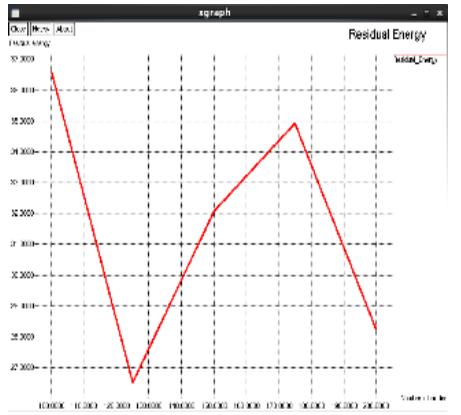

Fig. 8. Number of nodes vs. Residual energy 


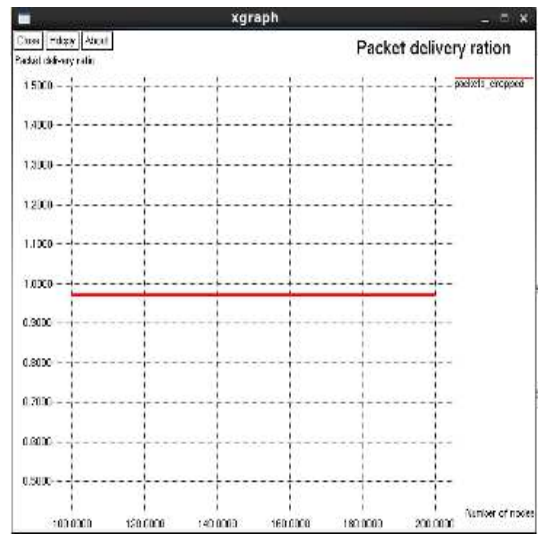

Fig. 9. Number of nodes vs. Packet delivery ratio

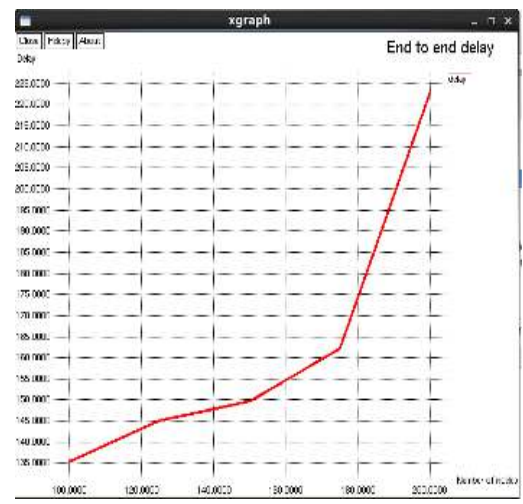

Fig. 10. Number of nodes vs. End to end delay

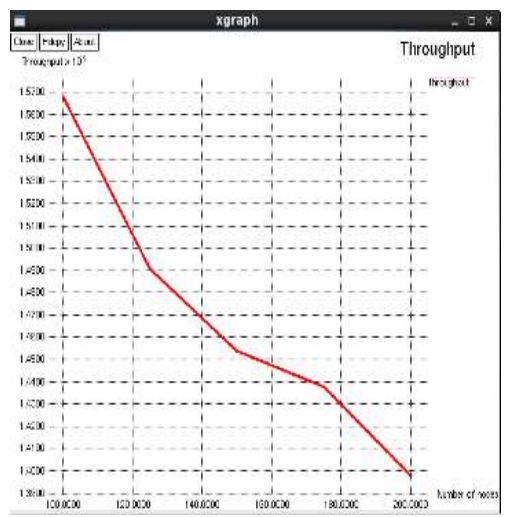

Fig 4.5 Number of nodes vs. Throughput 
The results of the simulation are transformed to graphical representation. The fig. 8 shows that residual energy varies greatly with increase in number of node. But packet delivery ratio remains same throughout as in Fig. 9. The end to end delay increases as shown in fig. 10. This shows that there is a packet drop when the node density increases resulting in delay. The throughput as shown in Fig 11 decreases with increase in node density. Thus the performance of AOMDV protocol dramatically decreases as the number of nodes increases.

\section{Conclusion And Future Work}

The performance of the routing protocol AOMDV deployed over MANET is studied in this research work using four parameters: end to end delay, packet delivery ratio, residual energy, and throughput. The simulations are carried out varying the node density and the results showed that selection of accurate properties depending upon the requirement that ultimately influences the efficiency of that network. The future work suggested is the development of a modified version of AOMDV routing protocols which should consider the energy of each node while transmitting packets. Thus making the protocol energy efficient AOMDV.

\section{References}

[1] Mahesh K. Marina and Samir R. Das, Ad hoc on-demand multipath distance vector routing, Wireless Communications And Mobile Computing 2006; 6:969-988

[2] Asis Nasipuri, Robert Castaneda, Samir R. Das, "Performance of muthipath routing for on-demand protocols in MANET", Mobile networks and Application, 2000

[3] MarjanRadi, "Multipath routing in WSN: Survey and research challenges", in Sensors, 2012

[4] Pankaj rohal, ruchikadahiya, prashantdahiya, "Study and analysis of throughput, delay and packet delivery ratio in MANET for topology based routing protocols", international journal for advance research in engineering and technology

[5] https://github.com/ebine/IEEE802.11-MM-Ns2-Simulation

[6] The network simulator - ns-2.29, http://www.isi.edu/nsnam/ns/, last accessed on Sep 2005.

[7] wpage.unina.it/marcello.caleffi/ns2/aomdv-old.html

[8] www.xgraph.prg/\#anch9 